\title{
Measurement of the radius of metallic plates based on a novel finite region eigenfunction expansion (FREE) method
}

\author{
Ruochen Huang, Mingyang Lu, Wuliang Yin*
}

\begin{abstract}
Eddy current based approaches have been investigated for a wide range of inspection applications. DoddDeeds model and the truncated region eigenfunction expansion (TREE) method are widely applied in various occasions, mostly for the cases that the sample is relatively larger than the radius of the sensor coil. The TREE method converts the integral expressions to the summation of many terms in the truncated region. In a recent work, the impedance of the co-axial air-cored sensor due to a plate of finite radius was calculated by the modified Dodd-Deeds analytical approach proposed by authors. In this paper, combining the modified analytical solution and the TREE method, a new finite region eigenfunction expansion (FREE) method is proposed. This method involves modifying its initial summation point from the first zero of the Bessel function to a value related to the radius of the plate, therefore makes it suitable for plate with finite dimensions. Experiments and simulations have been carried out and compared for the verification of the proposed method. Further, the planar size measurements of the metallic circular plate can be achieved by utilising the measured peak frequency feature.
\end{abstract}

Index Terms-Non-destructive testing, finite region eigenfunction expansion (FREE) method, finite dimension, magnetic induction, size measurements

\section{INTRODUCTION}

$\mathrm{E}$ DDY current testing has a promising prospect to inspect the quality of various materials, which has been successfully applied in the diverse fields of non-destructive testing (NDT), such as crack detection and orientation measurement by the sensor system with a rotating field [1-2], and the inversion of the thickness, conductivity and permeability of metal plate from the eddy current data [3-27]. Many researches have carried out investigations by using the single / multi-frequency eddy current testing and the pulsed eddy current (PEC) testing techniques, both of which are commonly applied and can obtain good results with high detection accuracy.

Pulsed eddy current testing, as a time domain method, injects a rectangular stimulus signal into the excitation coil during testing. Using the three significant features of the pulsed eddy current signal, the characteristic information of samples can be

This research was sponsored by Shanxi International Science and Technology Cooperation Projects (Grant No. 201803D421038).

R. Huang, M. Lu, and W. Yin are with the School of Electrical and Electronic Engineering, University of Manchester, Sackville Street Building, derived [28-31]. The signal from a differential-pulsed eddy current probe was used to evaluate the thickness of the metal plate in [32]. However, there was a trade-off between the peak value feature and the other two features. The use of the PEC techniques in eliminating the lift-off effect has also been developed [33-34].

In recent decades, the potential of multi-frequency eddy current techniques has also been explored [35-39]. Combining the experimental measurements and the digital data processing by utilising the linear combination of Walsh functions, the experimental method proposed by Egorov et al. provides high accuracy and reliable classification of the material properties in the process of aluminium alloy testing [35]. Dodd-Deeds model [41] and truncated region eigenfunction expansion (TREE) method [42] play a crucial role as the theoretical basis for the studies. Yin et al. discovered the relationship between the peak frequency and thickness / conductivity of the sample plate by using the Dodd-Deeds model [40]. A novel triple planar coil was been designed for the thickness measurement in [39]. The proposed algorithm used single frequency measurement approach and could not only improve the testing efficiency but also was immune to the lift-off effect. Instead of the integral expressions, the series one by using TREE method presented by $\mathrm{Li}$ and Theodoulidis had a good performance for the computation of magnetic field of the multilayered specimens with higher computational efficiency [43]. Furthermore, with the aid of the TREE method, Tytko et al. proposed a computation method suitable for both the E-cored coil with the circular air gap and the air gap of I-cored coil [44]. However, few analytical methods could be applied to predict the radius of circular plates with finite size.

Previously, in order to address the issue of the measurements caused by small sample plates, that is, the radius of the sample plates is smaller or near to that of sensor coils, the modified analytical solution was presented [45]. In this paper, based on the modified analytical solution and TREE method, a new method - termed as the finite region eigenvalue expansion (FREE) method is proposed for this case. Instead of summing all the eigenvalues from the boundary condition, a truncated series is extracted for the summation. Therefore, the impedance

Manchester M13 9PL, United Kingdom (Corresponding author: W. Yin, email: wuliang.yin@manchester.ac.uk). 
of a co-axial air-cored sensor above a plate of finite radius can be calculated. This method can relieve significant computation burden and potentially be applied for the real-time measurement. The results of simulations and experiments are compared and discussed. Moreover, the planar size of the circular disk plate samples can be estimated by comparing the measurement results and the modelling results using the proposed method.

\section{Methodology}

\section{A. The modified analytical solution}

In the traditional techniques of the magnetic induction and eddy current testing, one of the most frequently used tools is the coil impedance. Combining measurements and theoretical analysis, the features of the samples can be estimated from the received signals as the probe scanning across the sample plates. As the fundamental basis of the magnetic induction for the cylindrical coil, Dodd-Deeds model provides a solid theoretical background for the testing process. However, Dodd-Deeds analytical solution provides the models for infinite large plate, instead of the finite ones. The modified analytical solution based on Dodd and Deeds analytical solution [45] was proposed to address the issue when the inspected samples could not satisfy the condition - i.e. infinite planar size.

As shown in Fig. 1, the integral range of the original DoddDeeds analytical solution is from 0 to infinity. However, in the modified analytical solution, with the aid of the initial integrating point $\left(\alpha_{r_{s}}\right)$, the impedance of the coil above a metallic plate with a finite planar size can be calculated. It has been found that the value of $\alpha$ is inversely related to the planar dimension (i.e. radius) of the sample plates. For example, for the plate with infinite planar boundary (as shown in Fig. 1), $\alpha=$ 0 corresponds to the plate section of infinite radius while $\alpha=$ $\infty$ corresponds to the center of the sample plate.

Therefore, the vector potential induced by the excitation coil above the finite-sized sample plate, as shown in Fig. 2, can be expressed as the following [45],

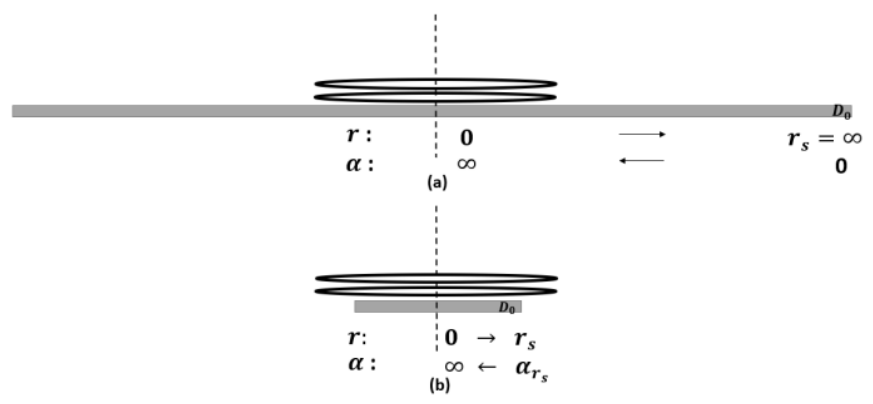

Fig. 1. Illustration of the integrational path for the modified analytical solution

$$
\mathrm{A}(\mathrm{r}, \mathrm{z})=\frac{\mathrm{I} \mu_{0} \mathrm{~N}_{\mathrm{e}}}{2} \int_{\alpha_{r_{s}}}^{\infty} \frac{\mathrm{J}_{1}(\alpha \mathrm{r}) \mathrm{P}(\alpha)}{\alpha^{3}} \mathrm{~K}(\mathrm{r}, \mathrm{z}, \alpha) \phi(\alpha) \mathrm{d} \alpha
$$

where

$$
\begin{gathered}
\phi(\alpha)=\frac{\left(\alpha_{1}+\mu_{1} \alpha\right)\left(\alpha_{1}-\mu_{1} \alpha\right) \mathrm{e}^{-2 \alpha_{1} \mathrm{D}_{0}}-\left(\alpha_{1}+\mu_{1} \alpha\right)\left(\alpha_{1}-\mu_{1} \alpha\right)}{-\left(\alpha_{1}-\mu_{1} \alpha\right)\left(\alpha_{1}-\mu_{1} \alpha\right) \mathrm{e}^{-2 \alpha_{1} \mathrm{D}_{0}}+\left(\alpha_{1}+\mu_{1} \alpha\right)\left(\alpha_{1}+\mu_{1} \alpha\right)} \\
\alpha_{1}=\sqrt{\alpha^{2}+\mathrm{j} \omega \sigma \mu_{1} \mu_{0}} \\
\mathrm{~K}(\mathrm{r}, \mathrm{z}, \alpha)=2-\mathrm{e}^{-\alpha\left(\mathrm{z}-\mathrm{l}_{\mathrm{e} 2}\right)}-\mathrm{e}^{-\alpha\left(\mathrm{z}-\mathrm{l}_{\mathrm{e} 1}\right)}+\mathrm{e}^{-\alpha \mathrm{z}}\left(\mathrm{e}^{-\alpha \mathrm{l}_{\mathrm{e} 1}}-\mathrm{e}^{-\alpha \mathrm{l}_{\mathrm{e}} 2}\right)
\end{gathered}
$$

$$
P(\alpha)=\int_{\alpha \mathrm{r}_{\mathrm{e} 1}}^{\alpha \mathrm{r}_{\mathrm{e} 2}} \tau \mathrm{J}_{1}(\tau) \mathrm{d} \tau
$$

Then, the change of the impedance due to the sample plate (after subtracting the impedance in the free space) is

$$
\begin{aligned}
& \Delta \mathrm{Z}(\omega)=\frac{\left.\mathrm{V}\right|_{\sigma, \mu_{1}}-\left.V\right|_{\sigma=0, \mu_{0}}}{\mathrm{I}}=\frac{\left.\mathrm{N}_{\mathrm{v}} \mathrm{j} \omega \int_{\mathrm{s}} \mathrm{Ads}\right|_{\sigma, \mu_{1}}-\left.\mathrm{N}_{\mathrm{v}} \mathrm{j} \omega \int_{\mathrm{s}} \mathrm{Ads}\right|_{\sigma=0, \mu_{0}}}{\mathrm{I}} \\
& =\frac{\mathrm{j} \omega \pi \mathrm{r}_{0} \mathrm{~N}_{\mathrm{v}} \mathrm{N}_{\mathrm{e}} \mu_{0}}{\left(\mathrm{I}_{\mathrm{v} 1}-\mathrm{l}_{\mathrm{v} 2}\right)^{2}\left(\mathrm{r}_{\mathrm{v} 1}-\mathrm{r}_{\mathrm{v} 2}\right)^{2}} \int_{\alpha_{\mathrm{r}_{\mathrm{s}}}}^{\infty} \frac{\mathrm{P}^{2}(\alpha)}{\alpha^{6}} \mathrm{e}^{-\alpha\left(2 l+\mathrm{l}_{\mathrm{e} 2}-\mathrm{l}_{\mathrm{e} 1}+\mathrm{g}\right)}(1 \\
& \left.-\mathrm{e}^{-2 \alpha\left(\mathrm{l}_{\mathrm{e} 2}-\mathrm{l}_{\mathrm{e} 1}\right)}\right) \phi(\alpha) \mathrm{d} \alpha \\
& \text { with } \alpha_{\mathrm{r}_{s}}=\frac{3.518}{\mathrm{r}_{s}}(3.518 \text { is the solution of a Bessel function. } \\
& \text { Details can be found in [45].) }
\end{aligned}
$$

Here, $\mathrm{N}_{\mathrm{e}}$ represents the number of turns of the transmitter; $\mathrm{N}_{\mathrm{v}}$ represents the number of turns of the receiver; $\mathrm{r}_{\mathrm{e} 1}$ and $\mathrm{r}_{\mathrm{v} 1}$ represent the inner radii of the transmitter and the receiver; $r_{\mathrm{e} 2}$ and $\mathrm{r}_{\mathrm{v} 2}$ represent the outer radii of the transmitter and the receiver; $\mathrm{l}_{\mathrm{e} 1}$ and $\mathrm{l}_{\mathrm{v} 1}$ represent the lower height of the transmitter and the receiver; $l_{\mathrm{e} 2}$ and $\mathrm{l}_{\mathrm{v} 2}$ represent the upper height of the transmitter and the receiver; $D_{0}$ represents the thickness of the tested sample; $\mu_{1}$ represents the relative permeability of the tested sample; $\sigma$ represents the conductivity of the tested sample; $\mu_{0}$ represents the permeability of air; $l$ represents the lift-off of the sensor; g represents the gap between the transmitter and the receiver; $J_{1}$ denotes a first order Bessel function of the first kind; $\alpha$ and $\tau$ are the integration variables.

An important indicator, $\Delta K$, is defined as the ratio of the coil mutual impedance and the frequency, expressed as

$$
\begin{gathered}
\Delta K(\omega)=\frac{j \pi r_{0} N_{v} N_{e} \mu_{0}}{\left(l_{v 1}-l_{v 2}\right)^{2}\left(r_{v 1}-r_{v 2}\right)^{2}} \int_{\alpha_{\mathrm{r}_{s}}}^{\infty} \frac{\mathrm{P}^{2}(\alpha)}{\alpha^{6}} e^{-\alpha\left(2 l+l_{\mathrm{e} 2}-l_{\mathrm{e} 1}+\mathrm{g}\right)}(1 \\
\left.-\mathrm{e}^{-2 \alpha\left(\mathrm{l}_{\mathrm{e} 2}-\mathrm{l}_{\mathrm{e} 1}\right)}\right) \phi(\alpha) \mathrm{d} \alpha
\end{gathered}
$$

The purpose of using $\Delta K$ is to mitigate the impact of the excitation frequency so that it can intuitively illustrate the

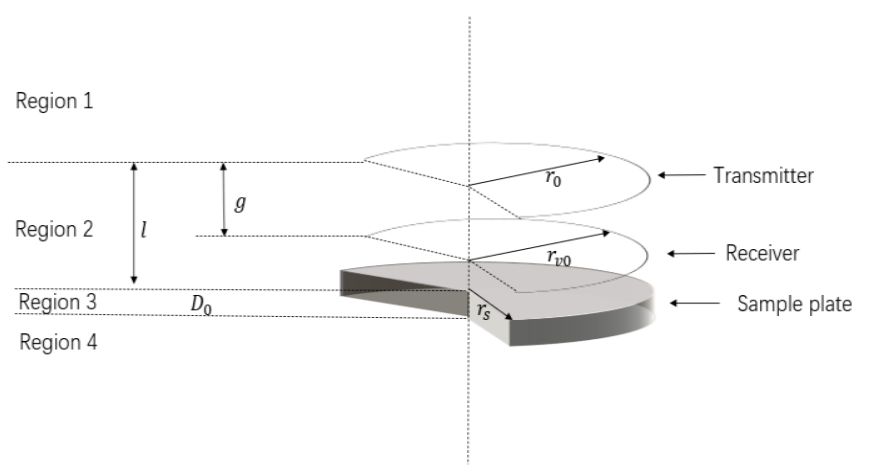

Fig. 2. The coil above the finite sample plate

characteristics caused by the properties of the sample plate. There exists a frequency feature (peak frequency) - the frequency when the real part of $\Delta \mathrm{K}$ reaches its peak value, which significantly depends on the conductivity and thickness of the plate.

\section{B. FREE modelling}

The TREE method has become a common analytical tool for the calculation of the eddy current problems, e.g. calculating the impedance of the coil probe above the testing plates. By replacing the integration expressions with the summation terms, the problem is simplified with suitable boundary conditions. As 
shown in Fig. 3(a), assuming that the region is truncated at h, which is normally is at least several times larger than the coil radius, Dirichlet boundary condition can be applied as

$$
J_{1}\left(\lambda_{i} h\right)=0
$$

The eigenvalues of equation (8) obtained from the boundary condition can be used for the numerical calculation for the solution domain of $\mathrm{r}=\mathrm{h}$. The impedance generated is given by

$$
\begin{aligned}
& \left.\Delta \mathrm{Z}(\omega)\right|_{r=h} \\
& =\frac{\mathrm{j} \omega \pi \mathrm{r}_{0} \mathrm{~N}_{\mathrm{v}} \mathrm{N}_{\mathrm{e}} \mu_{0}}{\left(\mathrm{l}_{\mathrm{v} 1}-\mathrm{l}_{\mathrm{v} 2}\right)^{2}\left(\mathrm{r}_{\mathrm{v} 1}-\mathrm{r}_{\mathrm{v} 2}\right)^{2}} \sum_{i=1}^{\infty} \frac{\mathrm{P}^{2}\left(\lambda_{i}\right)}{\lambda_{i}{ }^{6}} \mathrm{e}^{-\lambda_{i}\left(2 l+\mathrm{l}_{\mathrm{e} 2}-\mathrm{l}_{\mathrm{e} 1}+\mathrm{g}\right)}(1 \\
& \left.-\mathrm{e}^{-2 \lambda_{i}\left(\mathrm{l}_{\mathrm{e} 2}-\mathrm{l}_{\mathrm{e} 1}\right)}\right) \phi\left(\lambda_{i}\right)
\end{aligned}
$$

When the solution domain shrinks to $r_{s}$ (as shown in Fig. 3(b)), according to the modified analytical solution [45], the value of $\lambda$ is closely associated with the radius of the sample plates. Therefore, the terms of the summation expressions are truncated and now only range from $\lambda_{n}$ to infinity. The change of impedance of the sensor due to the presence of the sample plate should be

$$
\begin{aligned}
& \left.\Delta \mathrm{Z}(\omega)\right|_{r=r_{s}} \\
& =\frac{\mathrm{j} \omega \pi \mathrm{r}_{0} \mathrm{~N}_{\mathrm{v}} \mathrm{N}_{\mathrm{e}} \mu_{0}}{\left(\mathrm{l}_{\mathrm{v} 1}-\mathrm{l}_{\mathrm{v} 2}\right)^{2}\left(\mathrm{r}_{\mathrm{v} 1}-\mathrm{r}_{\mathrm{v} 2}\right)^{2}} \sum_{i=n}^{\infty} \frac{\mathrm{P}^{2}\left(\lambda_{i}\right)}{\lambda_{i}^{6}} \mathrm{e}^{-\lambda_{i}\left(2 l+\mathrm{l}_{\mathrm{e} 2}-\mathrm{l}_{\mathrm{e} 1}+\mathrm{g}\right)}(1 \\
& \left.-\mathrm{e}^{-2 \lambda_{i}\left(\mathrm{l}_{\mathrm{e} 2}-\mathrm{l}_{\mathrm{e} 1}\right)}\right) \phi\left(\lambda_{i}\right)
\end{aligned}
$$

Where $\lambda_{n}$ is the smallest solution (eigenvalues) of (8) that satisfies $\lambda_{n} \geq \alpha_{r_{s}}$.

Further, the indicator $\Delta K$ due to the presence of the finite size plate can be represented as

$$
\begin{aligned}
& \left.\Delta \mathrm{K}(\omega)\right|_{r=r_{s}} \\
& =\frac{\mathrm{j} \pi \mathrm{r}_{0} \mathrm{~N}_{\mathrm{v}} \mathrm{N}_{\mathrm{e}} \mu_{0}}{\left(\mathrm{l}_{\mathrm{v} 1}-\mathrm{l}_{\mathrm{v} 2}\right)^{2}\left(\mathrm{r}_{\mathrm{v} 1}-\mathrm{r}_{\mathrm{v} 2}\right)^{2}} \sum_{i=n}^{\infty} \frac{\mathrm{P}^{2}\left(\lambda_{i}\right)}{\lambda_{i}{ }^{6}} \mathrm{e}^{-\lambda_{i}\left(2 l+\mathrm{l}_{\mathrm{e} 2}-\mathrm{l}_{\mathrm{e} 1}+\mathrm{g}\right)}(1 \\
& \left.-\mathrm{e}^{-2 \lambda_{i}\left(1_{\mathrm{e} 2}-\mathrm{l}_{\mathrm{e} 1}\right)}\right) \phi\left(\lambda_{i}\right)
\end{aligned}
$$

\section{VERIFICATION}

\section{A. Experimental setup}

As can be seen from Fig. 4, in order to measure the impedance of sensor probe due to the presence of the circular planar plate, an air-cored sensor probe was designed, with the transmitter, the receiver coil and the non-magnetic metallic sample plate coaxially placed. The transmitter was located above the receiver and the geometry profile of the sensor is listed in Table I. In the

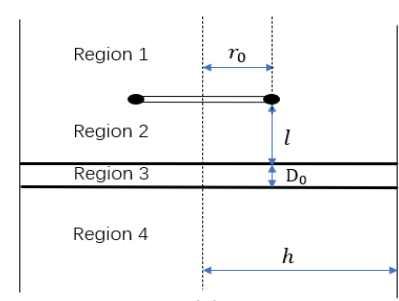

(a)

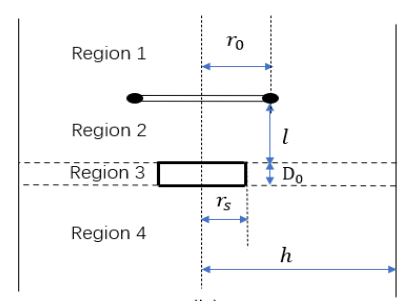

(b)

Fig. 3. Truncated region (a) the solution domain $r=h$, (b) the solution domain $r=r_{s}$.

measurements, the transmitter and the receiver had the same number of turns (15 turns) with the inner radius of $28 \mathrm{~mm}$ and the outer radius of $28.25 \mathrm{~mm}$. The gap between the transmitter and the receiver was set to $15 \mathrm{~mm}$. Copper and aluminium plates, whose conductivities are $57 \mathrm{MS} / \mathrm{m}$ and $35 \mathrm{MS} / \mathrm{m}$

respectively, were chosen as the tested samples. A series of copper plate size were used to verify the proposed method, which ranged from $25 \mathrm{~mm}$ to $45 \mathrm{~mm}$ with the same thickness $(1 \mathrm{~mm})$, while the aluminium plates had an identical size (the diameter of $40 \mathrm{~mm})$ but different thicknesses $(22 \mu \mathrm{m}$ and 66 $\mu \mathrm{m})$. Multifrequency mode was used in the measurement. As shown in Fig. 5, the Zurich impedance analyser was used to measure the mutual impedance of the sensor-sample system. The measured impedance $-Z_{\text {sample }}$, was subtracted by that in the free space $-Z_{\text {air }}$. The instrument can operate up to a higher excitation frequency of $510 \mathrm{kHz}$ with a high accuracy. Equations (12) - (13) demonstrate the data processing for the recorded signal from the instruments.

$$
\begin{aligned}
\Delta \mathrm{Z}(\omega) & =Z_{\text {sample }}(\omega)-Z_{\text {air }}(\omega) \\
\Delta \mathrm{K}(\omega) & =\frac{Z_{\text {sample }}(\omega)-Z_{\text {air }}(\omega)}{\omega}
\end{aligned}
$$

TABLE I

SENSOR PROPERTIES

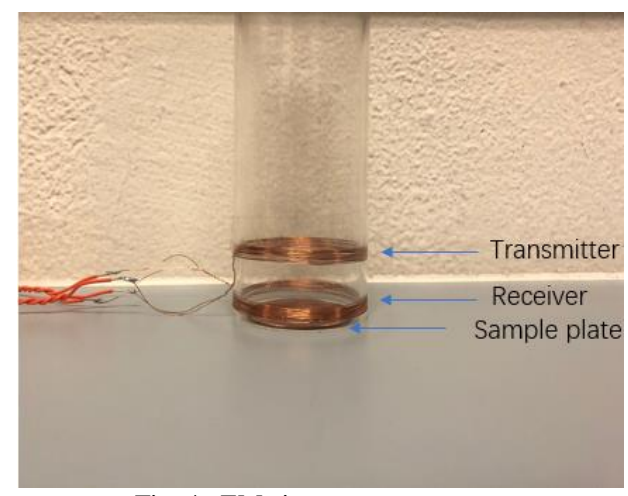

Fig. 4. EM air-core sensor setup.

\begin{tabular}{ll}
\hline \hline $\mathrm{r}_{\mathrm{x} 1}$ (Inner radii of the transmitter / & $28 \mathrm{~mm}$ \\
the receiver) & \\
$\mathrm{r}_{\mathrm{x} 2}$ (Outer radii of the transmitter & $28.25 \mathrm{~mm}$ \\
/ the receiver) & \\
$\mathrm{l}_{\mathrm{e} 2}-\mathrm{l}_{\mathrm{e} 1}$ (Height of the transmitter) & $4 \mathrm{~mm}$ \\
$\mathrm{l}_{\mathrm{v} 2}-\mathrm{l}_{\mathrm{v} 1}$ (Height of the receiver) & $4 \mathrm{~mm}$ \\
$\mathrm{~N}_{x}$ (Number of turns of the & 15 \\
transmitter / the receiver) & $1 \mathrm{~mm}$ for the copper, $22 \mu \mathrm{m}$ \\
$D_{0}$ (Sample thickness) & and $66 \mu \mathrm{m}$ for the aluminium \\
& $25 \mathrm{~mm}, 30 \mathrm{~mm}, 35 \mathrm{~mm}$, \\
$d_{S}$ (diameter of sample plate & $40 \mathrm{~mm}$ and $45 \mathrm{~mm}$ for the \\
size) & copper, $40 \mathrm{~mm}$ for the \\
& aluminium \\
$\mathrm{g}$ (The coil separation) & $15 \mathrm{~mm}$
\end{tabular}




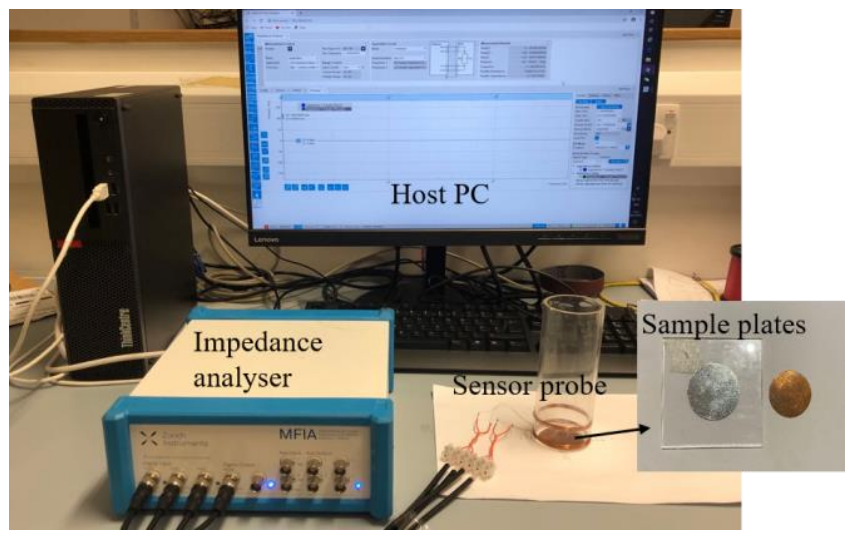

Fig. 5. Measurement setup.

\section{B. FEM simulation}

In addition to the experimental measurement, the eddy current problems were also investigated by the finite element method (FEM). In this paper, FEM simulation was carried out to investigate the validity of the proposed FREE method. In the FEM simulation, magnetic insulation boundary condition was used. The excitation current was set to $1 \mathrm{~A}$ and the impedance due to the presence of the sample plate and the impedance without the existence of the sample plate were calculated, then, the impedance change due to the sample plate can be obtained. Two-dimensional axisymmetric models in COMSOL were created to compare with the proposed FREE method and verify the accuracy of the method. Since the precision of the FEM method highly depends on the discretisation of the entire mesh model, dense mesh was selected for the aluminium plates. The discretised mesh model for the aluminium plate with a thickness of $22 \mu \mathrm{m}$ plate is shown in Fig. 6 .

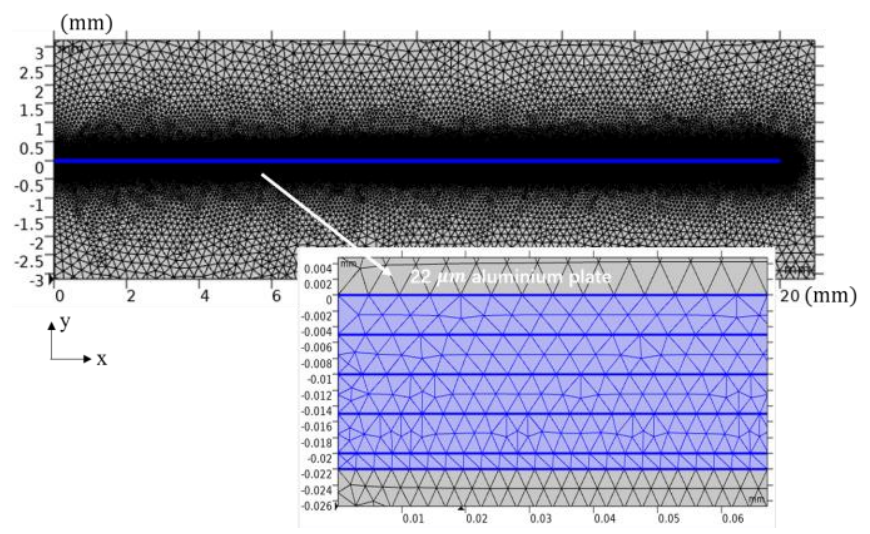

Fig. 6. Mesh model for the aluminium plate with a thickness of $22 \mu \mathrm{m}$.

\section{Results}

1) Verification of the FREE method

Figures 7, 8 and 9 demonstrate both the real part and the imaginary part of the analytical solutions, simulations and measurements of the testing system in multi-frequency mode under two different thicknesses of the aluminium plates. In [45], the initial summation point is related with the radius of the sample, which is immune to the sample thickness. Hence, the same initial summation point has been used in the method.
As depicted in Fig. 8 and 9, the magnitude of $\Delta \mathrm{K}$ reduces compared with the sample with infinite radius. And at the same time, the frequency feature (peak frequency) in the real part of $\Delta \mathrm{K}$ changes significantly - increases with the decreased radius of the sample plate.

From Fig. 8 and 9, it can be clearly seen that FREE method shows a good agreement to the other methods. The peak frequency in the real part of $\Delta \mathrm{K}$ shifts leftwards as the thickness of the sample increases. It corresponds to the phenomenon investigated in [40]. There is a small discrepancy, particularly under high frequency, among these methods, as can be seen from Fig. 8(a) and Fig. 9(a). The reason for the small discrepancy may be that to achieve high accuracy in FEM simulation, the mesh has to be generated rather dense, however, the size of the entire mesh domain (including surrounding free space) is limited due to constraints in computation power for the solver, which causes certain error. In the measurements, the slight axis displacement between the probe and the sample plate can also cause error. However, as shown in Table II, the FREE method is much more efficient than the FEM simulations.

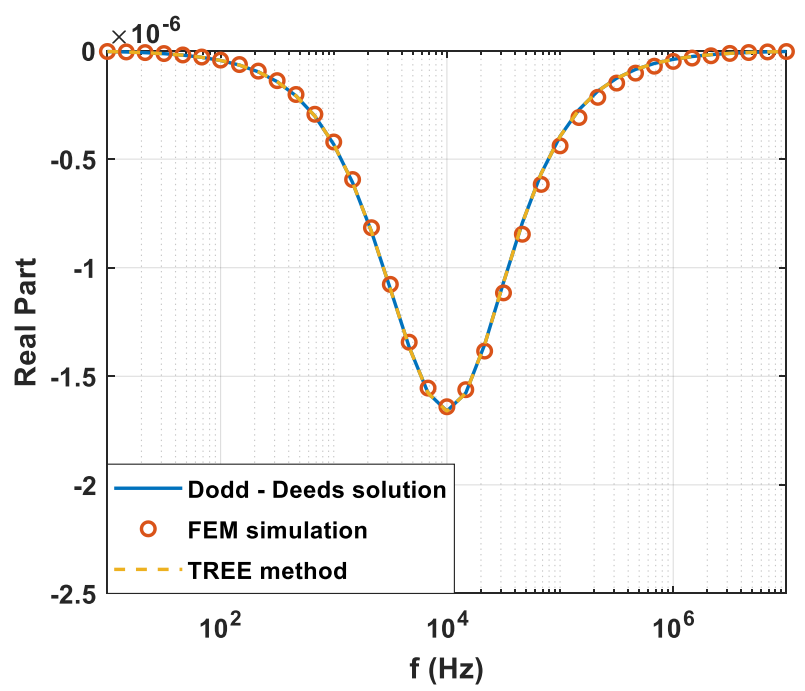

(a)

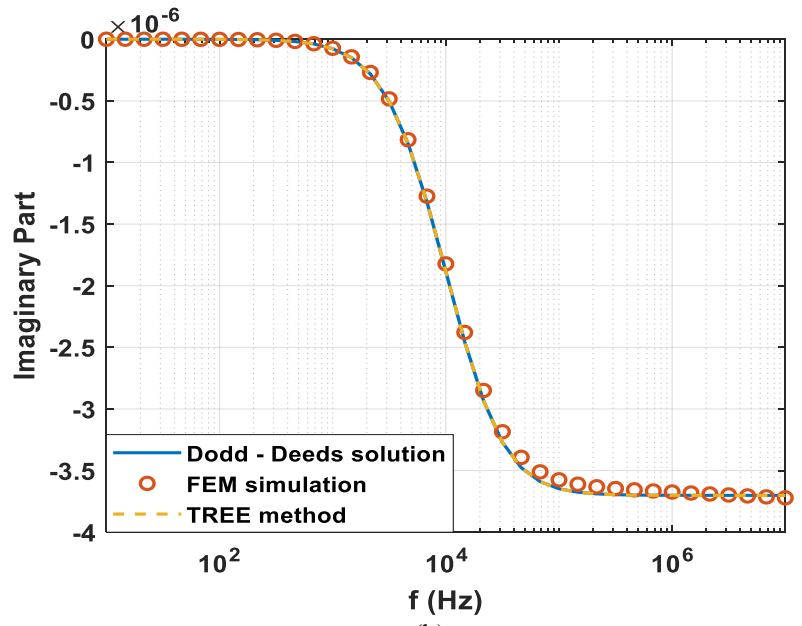

(b)

Fig. 7. The change of the indicator $\Delta K$ for the infinite aluminium plate with the thickness of $22 \mu \mathrm{m}$ (a) real part (b) imaginary part 


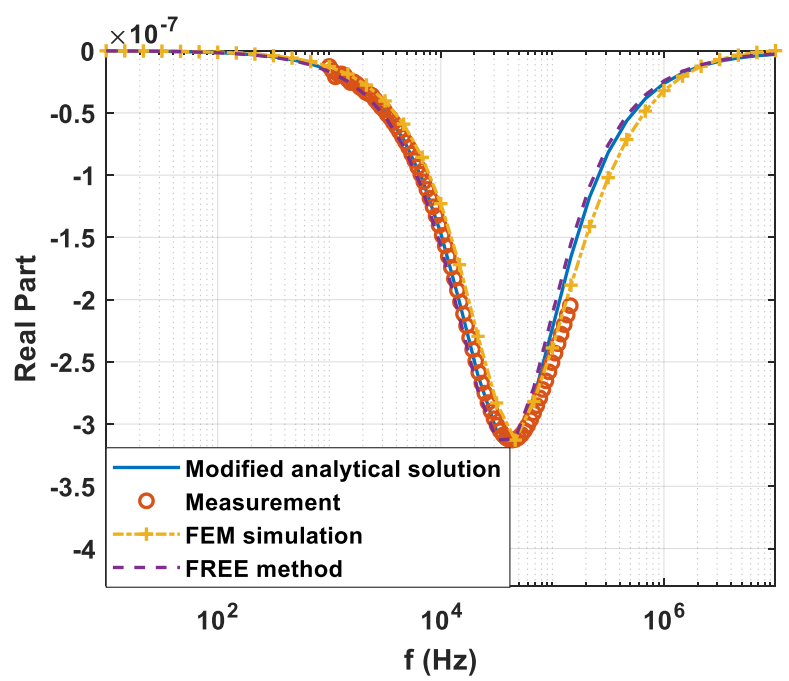

(a)

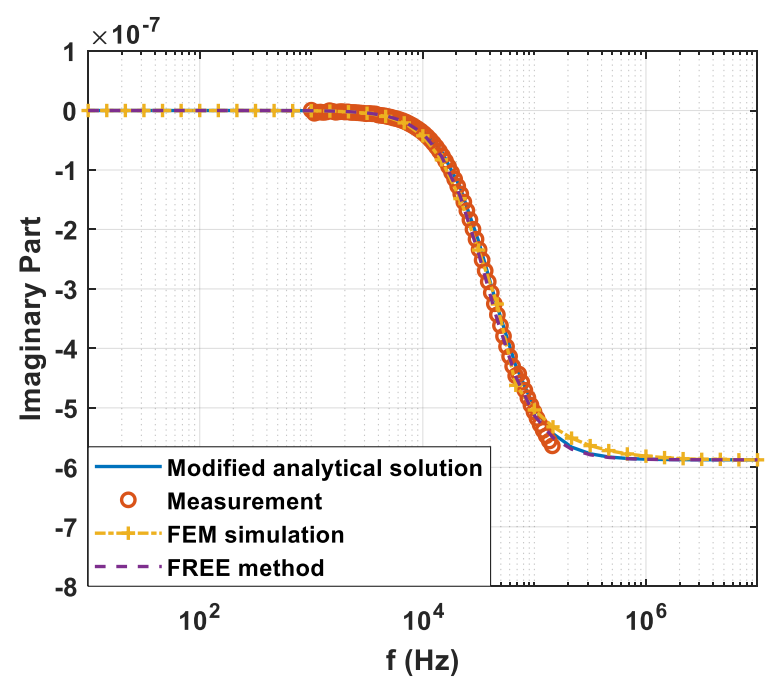

(b)

Fig. 8. The change of the indicator $\Delta \mathrm{K}$ for the aluminium plate with the thickness of $22 \mu \mathrm{m}$ and the radius of $20 \mathrm{~mm}$ (a) real part (b) imaginary part

TABLE II

Running Time of FREE Method and FEM Simulation

\begin{tabular}{ccccc}
\hline \hline Material & $\begin{array}{c}\text { Thickness } \\
(\mu \mathrm{m})\end{array}$ & $\begin{array}{c}\text { Frequency } \\
\text { range }(\mathrm{Hz})\end{array}$ & \multicolumn{2}{c}{ Time (s) } \\
\cline { 4 - 5 } & 22 & $10-10^{7}$ & $\begin{array}{c}\text { FREE } \\
\text { Method }\end{array}$ & $\begin{array}{c}\text { FEM } \\
\text { Simulation }\end{array}$ \\
\hline Aluminium & 66 & $10-10^{7}$ & 0.54 & 31 \\
& 6 & & & 50 \\
\hline
\end{tabular}

\section{2) Measurement of the plate planar size based on the FREE method}

In principle, by referring to (6) and (10), the relationship between the value of the initial summation point and the radius of the sample plate can be obtained. Further experiments have been carried out to verify this relationship. That is, by matching with the real part of the measured impedance curve in the multifrequency spectral, the initial summation point from the eigenvalues of the Bessel function can be approximated. Consequently, the planar size of the plate can be reconstructed. In order to validate the proposed plate size reconstruction technique, the impedance change of the sensor-sample system due to copper plates with varying size (shown in Table I) have

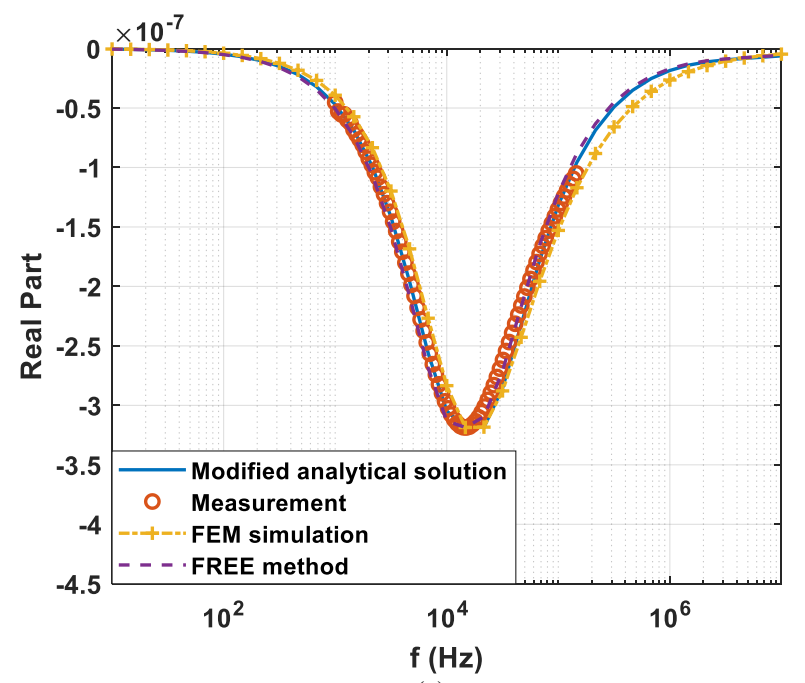

(a)

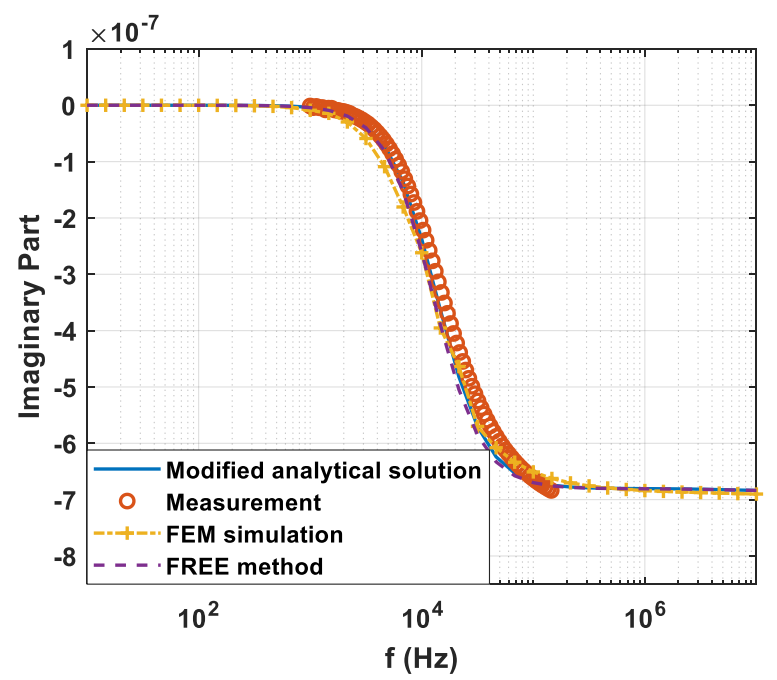

(b)

Fig. 9. The change of the indicator $\Delta \mathrm{K}$ for the aluminium plate with the thickness of $66 \mu \mathrm{m}$ and the radius of $20 \mathrm{~mm}$ (a) real part (b) imaginary part

been measured. The thicknesses of the copper samples are kept to $1 \mathrm{~mm}$ in the measurements.

Fig. 10 exhibits the measurements and the analytical solution (FREE method) of the impedance with copper plates of several radii. There is a discrepancy between two methods particularly under higher frequencies. The error incurred may be due to the skin depth effect. As known, the skin depth is inversely related to the conductivity of the sample plate. As expected, compared with the measurements for the aluminium sample plates, the skin effect has a greater impact on the copper plates. Besides, the deviation of the measurement under the high frequency maybe caused by the resonance effect of the coil and the stray capacitive /inductive elements introduced by the connecting cables during the measurement process. It can be observed that the magnitude of the impedance drops off with the decreased sample size. Besides, not only the thickness and the properties of the sample plate, but also the planar size of the sample plate affects the value of the frequency feature (peak frequency) - the frequency when real part of $\Delta \mathrm{K}$ reaches its peak value. That is, the larger the planar size, the smaller the frequency feature 
(peak frequency). For this measurement, the thicknesses of the sample plates were kept the same, hence the frequency shift is due to the radius of the sample plate. This is a limitation of this method, i.e. to reliably measure the sample radius its thickness needs to be known. For the size measurements, the key to obtain a good estimation is to find the peak frequency. The curve fitting method by using the polynomial function and bisquare weights was used to approximate the position of the peak frequency. A range of frequency results were extracted for the approximation. For the case of the sample with the radius of $12.5 \mathrm{~mm}$, the results can be approximated as the polynomial function, as shown in the following equation.

Where:

$$
y=a(\log f)^{4}+b(\log f)^{3}+c(\log f)^{2}+d \log f+e
$$

$a=-1.734 e-12 ; b=5.295 e-11 ; c=-5.935 e-10$;

$d=2.889 e-09 ; e=-5.171 e-09$.

Hence the peak frequency can be determined by the position of the minimum value which can be obtained by finding the stationary point for equation (14).

Figure 11 depicts the estimated initial summation point for the copper plates under varying radii. It can be concluded from Table III that, the reconstruction of the plate planar size shows

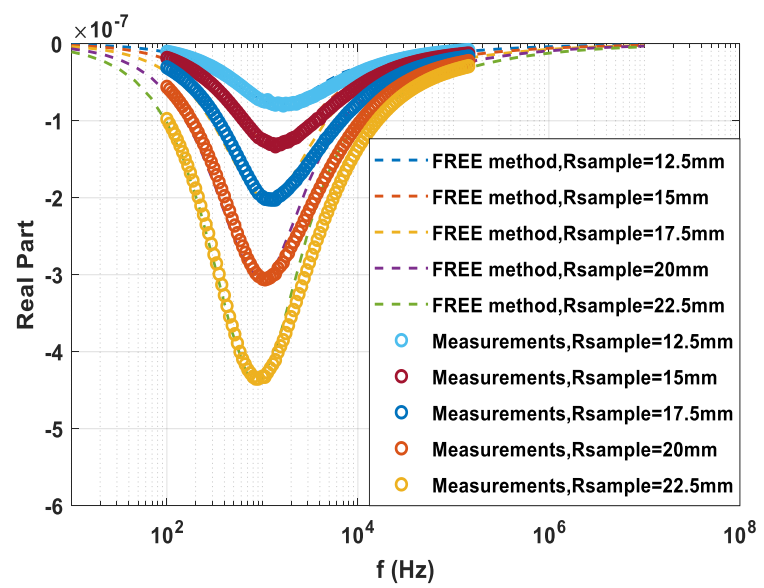

Fig. 10. The real part of $\Delta \mathrm{K}$ under several planar size of copper plates with the thickness of $1 \mathrm{~mm}$.

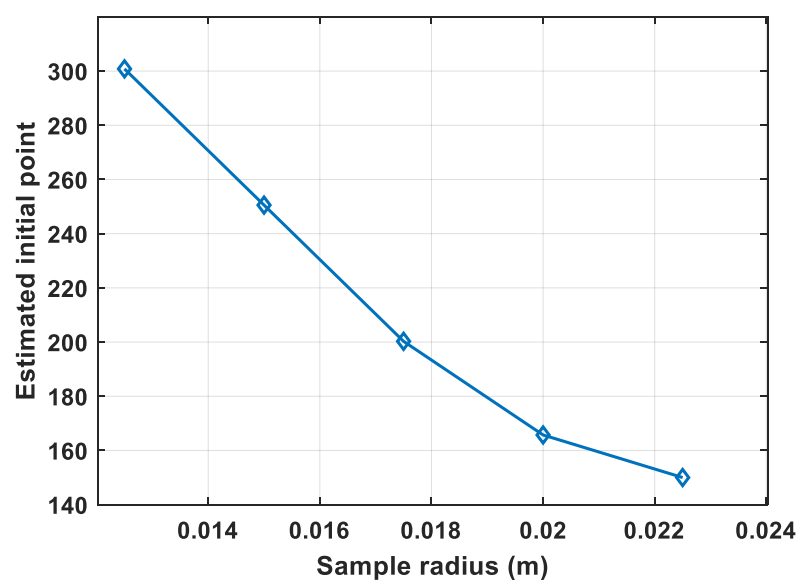

Fig. 11. The estimated initial summation points for copper plates in relation to sample radius a good performance via the proposed method, as the error of the measured size is limited within a reasonable value of $7 \%$.

TABLE III

Actual and Estimated Plate Planar Size for the Copper Plates

\begin{tabular}{cccc}
\hline \hline Material & $\begin{array}{c}\text { Actual radius } \\
(\mathrm{mm})\end{array}$ & $\begin{array}{c}\text { Estimated } \\
\text { radius }(\mathrm{mm})\end{array}$ & $\begin{array}{c}\text { Error } \\
(\%)\end{array}$ \\
\hline Copper & 12.5 & 11.6 & 6.9 \\
& 15 & 14.0 & 6.8 \\
& 17.5 & 17.3 & 1.4 \\
& 20 & 21.1 & 5.6 \\
& 22.5 & 23.3 & 3.7 \\
\hline
\end{tabular}

\section{CONCLUSIONS}

In this paper, a new method called finite region eigenfunction expansion (FREE) is proposed for the impedance computation for finite size metallic sample plate. With the aid of the FREE method, the planar size of the metallic plate can be reconstructed. The FREE method was developed by combining the modified Dodd-Deeds analytical solution and the TREE method. It is essentially an approximation of the first order. The modified Dodd-Deeds analytical solution introduced a new initial integration point, which is related to the radius of the sample plate. Extending this concept to the TREE method, a new initial summation point was introduced in the TREE method to tackle the case of finite domain of the plate especially when the radius of the sample is smaller than the sensor radius. Both measurements and FEM simulations have been carried out for the verification of the proposed FREE method. The results show a good agreement between them. In addition, the peak feature is used for size measurement, which is related to both plate thickness and the size. Hence, with the known thickness of the sample plate, the radius can be estimated with a small error value within $7 \%$. The error can be reduced by the setup of the measurements and the setting (i.e. frequency step) of the instrument.

\section{ACKNOWLEDGEMENT}

This research was sponsored by Shanxi International Science and Technology Cooperation Projects (Grant No. 201803D421038).

\section{REFERENCES}

[1]. G. Yang, G. Dib, L. Udpa, A. Tamburrino, and S. S. Udpa, "Rotating field EC-GMR sensor for crack detection at fastener site in layered structures," IEEE Sensors Journal, vol. 15, no. 1, pp. 463-470, Jan. 2015.

[2]. W. Li, X. Yuan, G. Chen, J. Ge, X. Yin and K. Li, "High sensitivity rotating alternating current field measurement for arbitrary-angle underwater cracks," NDT\&E International, vol. 79, pp. 123-131, Apr. 2016.

[3]. J. C. Moulder, E. Uzal, and J. H. Rose, "Thickness and conductivity of metallic layers from eddy current measurements," Review of Scientific Instruments, vol. 63, no. 6, 1992.

[4]. X. Chen and Y. Lei, "Electrical conductivity measurement of ferromagnetic metallic materials using pulsed eddy current method," NDT \& E International, vol. 75, pp. 33-38, 2015. 
[5]. W. Yin, S. J. Dickinson, and A. Peyton, "Imaging the continuous conductivity profile within layered metal structures using inductance spectroscopy," IEEE Sensors Journal, vol. 5, no. 2, pp. 161-166, 2005.

[6]. E. Pinotti and E. Puppin, "Simple lock-in technique for thickness measurement of metallic plates," IEEE Transactions on Instrumentation and Measurement, vol. 63, no. 2, pp. 479-484, Feb. 2014.

[7]. M. Lu, R. Huang, W. Yin, Q. Zhao, and A. Peyton, "Measurement of permeability for ferrous metallic plates using a novel lift-off compensation technique on phase signature," IEEE Sensors Journal, vol. 19, no. 17, pp. 7440-7446, 1 Sept.1, 2019

[8]. M. Lu, et al. "Determination of the magnetic permeability, electrical conductivity, and thickness of ferrite metallic plates using a multifrequency electromagnetic sensing system," IEEE Transactions on Industrial Informatics, vol. 15, pp. 4111-4119, 2019.

[9]. M. Lu, et al. "Acceleration of frequency sweeping in eddy-current computation," IEEE Transactions on Magnetics, vol. 53, pp. 1-8, 2017.

[10]. T. Yang, et al. "Level measurement for saline with a small surface area using high frequency electromagnetic sensing technique," Measurement, vol. 101, pp. 118-125, 2017.

[11]. W. Zhou, M. Lu et al. "Three-dimensional electromagnetic mixing models for dual-phase steel microstructures," Applied Sciences, vol. 8, pp. 529,2018

[12]. M. Lu et al. "Prediction of the asymptotical magnetic polarization tensors for cylindrical samples using the boundary element method," 2015 IEEE Sensors Applications Symposium (SAS), pp. 1-4, 2015.

[13]. W. Yin et al. "An equivalent-effect phenomenon in eddy current nondestructive testing of thin structures," IEEE ACCESS, vol. 7, pp. 70296 - 70307, 2019.

[14]. M. Lu et al. "Measurement of ferromagnetic slabs permeability based on a novel planar triple-coil sensor," IEEE Sensors J., vol. 20, no. 6, pp. 2904-2910, 2020.

[15]. J.R.S. Avila, M. Lu et al. "Accurate measurements of plate thickness with variable lift-off using a combined inductive and capacitive sensor," NDT \& E International, vol. 110, pp. 102202, 2020.

[16]. H. Xu, M. Lu et al. "Imaging Weld Cross-Section Using a Novel Frequency Feature in Multi-Frequency Eddy Current Testing," InsightNon-Destructive Testing and Condition Monitoring, vol. 61, pp. 738743, 2019.

[17]. W. Yin et al. "Permeability invariance phenomenon and measurement of electrical conductivity for ferrite metallic plates," Insight-NonDestructive Testing and Condition Monitoring, vol. 61, pp. 472-479, 2019.

[18]. L. Yin et al. "Detection of corrosion pits based on an analytically optimised eddy current sensor," Insight-Non-Destructive Testing and Condition Monitoring, vol. 60, pp. 561-567, 2018.

[19]. M. Lu et al. "Determining the magnetic permeability of ferrite steel strip by a custom inversion method," Proc. 12th ECNDT, pp. 1-8, 2018.

[20]. J. Tang et al. "Bio-impedance spectroscopy for frozen-thaw of biosamples: Non-contact inductive measurement and finite element (FE) based cell modelling," Journal of Food Engineering, vol. 272, pp. 109784, 2020.

[21]. R. Huang, M. Lu et al. "A novel perturbed matrix inversion based method for the acceleration of finite element analysis in crack-scanning eddy current NDT," IEEE Access, vol. 8, pp. 12438-12444, 2020.

[22]. W. Yin, M. Lu et al. "Custom edge-element FEM solver and its application to eddy-current simulation of realistic $2 \mathrm{M}$-element human brain phantom," Bioelectromagnetics, vol. 39, pp. 604-616, 2018.

[23]. W. Yin, M. Lu et al. "Acceleration of eddy current computation for scanning probes," Insight-Non-Destructive Testing and Condition Monitoring, vol. 60, pp. 547-555, 2018.

[24]. J. Tang et al. "A Novel Efficient FEM Thin Shell Model for BioImpedance Analysis," Biosensors, vol. 10, pp. 69, 2020.

[25]. Y. Xie, M. Lu et al. "Novel Wearable Sensors for Biomechanical Movement Monitoring Based on Electromagnetic Sensing Techniques," IEEE Sensors J., vol. 20, no. 2, pp. 1019-1027, 2019.

[26]. M. Lu et al. "Forward solver for deep earth exploration and induction logging using custom built Edge-Element FEM technique," Acta Geologica Sinica - English Edition, vol. 93, pp. 302-304, 2019.

[27]. M. Lu, H. Xu, W. Zhu, L. Yin et al. "Conductivity Lift-off Invariance and measurement of permeability for ferrite metallic plates," NDT \& E International, vol. 95, pp. 36-44, Apr. 2018.

[28]. A. Sophiana, G. Y. Tiana, D. Taylora, and J. Rudlinb, "A feature extraction technique based on principal component analysis for pulsed
Eddy current NDT," NDT \& E International, vol. 36, no. 1, pp. 37-41, Jan. 2003.

[29]. D. Vasic, V. Bilas and D. Ambrus, "Pulsed eddy-current nondestructive testing of ferromagnetic tubes," IEEE Transactions on Instrumentation and Measurement, vol. 53, no. 4, pp. 1289-1294, Aug. 2004.

[30]. I. Z. Abidin, C. Mandache, G. Y. Tian, and M. Morozov, "Pulsed eddy current testing with variable duty cycle on rivet joints," $N D T \& E$ International, vol. 42, no. 7, pp. 599-605, 2009.

[31]. Y. He, G. Tian, H. Zhang, M. Alamin, A. Simm, and P. Jackson, "Steel corrosion characterization using pulsed eddy current systems," IEEE Sensors Journal, vol. 12, no. 6, pp. 2113-2120, June 2012.

[32]. Y. Shin, D. Choi, Y. Kim, and S. Lee, "Signal characteristics of differential-pulsed eddy current sensors in the evaluation of plate thickness," NDT \& E International, vol. 42, no. 3, pp. 215-221, 2009.

[33]. D. Wen, M. Fan, B. Cao, B. Ye, and G. Tian, "Extraction of LOI Features from spectral pulsed eddy current signals for evaluation of ferromagnetic samples," IEEE Sensors Journal, vol. 19, no. 1, pp. 189-195, 1 Jan.1, 2019.

[34]. G. Y. Tian and A. Sophian, "Reduction of lift-off effects for pulsed eddy current NDT," NDT \& E International, vol. 38, no. 4, pp. 319-324, 2005.

[35]. A. V. Egorov, V. V. Polyakov, D. S. Salita, E. A. Kolubaev, et al., "Inspection of aluminum alloys by a multi-frequency eddy current method," Defence Technology, vol. 11, no. 2, pp. 99-103, 2015.

[36]. W. Yin and A.J. Peyton, "Thickness measurement of non-magnetic plates using multi-frequency eddy current sensors," NDT \& E International, vol. 40, no. 1, pp. 43-48, 2007.

[37]. M. Lu, W. Zhu, L. Yin, A. J. Peyton, W. Yin, and Z. Qu, "Reducing the lift-off effect on permeability measurement for magnetic plates from multifrequency induction data," IEEE Transactions on Instrumentation and Measurement, vol. 67, no. 1, pp. 167-174, Jan. 2018.

[38]. M. Lu, L. Yin, A. J. Peyton and W. Yin, "A novel compensation algorithm for thickness measurement immune to lift-off variations using eddy current method," IEEE Transactions on Instrumentation and Measurement, vol. 65, no. 12, pp. 2773-2779, Dec. 2016.

[39]. M. Lu, X. Meng, W. Yin, Z. Qu, F. Wu, J. Tang, et al., "Thickness measurement of non-magnetic steel plates using a novel planar triple-coil sensor," NDT \& E International, vol. 107, 2019.

[40]. W. Yin, A. J. Peyton and S. J. Dickinson, "Simultaneous measurement of distance and thickness of a thin metal plate with an electromagnetic sensor using a simplified model," IEEE Transactions on Instrumentation and Measurement, vol. 53, no. 4, pp. 1335-1338, Aug. 2004.

[41]. C. V. Dodd, and W. E. Deeds, "Analytical solutions to eddy - current probe - coil problems," Journal of applied physics, vol. 39, no. 6, pp. 2829-2838, 1968.

[42]. T. Theodoulidis and E. E. Kriezis, "Eddy current canonical problems (with applications to nondestructive evaluation)," Tech Science Press, 1st edition, Apr. 2006.

[43]. Y. Li, T. Theodoulidis and G. Y. Tian, "Magnetic field-based eddycurrent modeling for multilayered specimens," IEEE Transactions on Magnetics, vol. 43, no. 11, pp. 4010-4015, Nov. 2007.

[44]. G. Tytko and L. Dziczkowski, "E-Cored coil with a circular air gap inside the core column used in eddy current testing," IEEE Transactions on Magnetics, vol. 51, no. 9, pp. 1-4, Sept. 2015.

[45]. R. Huang, M. Lu, A. Peyton, and W. Yin, "Thickness measurement of metallic plates with finite planar dimension using eddy current method," IEEE Transactions on Instrumentation and Measurement, early access, 2020. Doi:10.1109/TIM.2020.2987413. 